\title{
JUSTICIA DISTRIBUTIVA GLOBAL E INMIGRACIÓN
}

\author{
Distributive global justice and immigration
}

Daniel Loewe*

Resumen. El artículo evalúa argumentos presentados a favor de la inmigración que se basan en exigencias normativas de la justicia distributiva: el argumento liberal igualitario, el argumento de compensación, el argumento de la posesión común de la tierra, y el argumento utilitarista. Se sostiene que la migración no es el método más efectivo para avanzar los fines de la justicia distributiva global y que, por lo tanto, estos enfoques tienen un alcance menor en tanto argumentos a favor de la migración, aunque muy relevante en determinadas circunstancias.

Palabras claves: inmigración, justicia distributiva global, igualitarismo, compensación, posesión común de la tierra, movilidad sin fronteras.

Abstract. The paper evaluates arguments presented in favor of immigration that are based on normative demands of distributive justice: the liberal egalitarian argument, the compensation argument, the argument of the common possession of the earth, and the utilitarian argument. It argues that migration is not the most effective way to achieve the goals of global distributive justice and that therefore these approaches have less to say in favor of migration, although they are very relevant in certain circumstances.

Keywords: immigration, distributive global justice, egalitarianism, compensation, common possession of the earth, open borders.

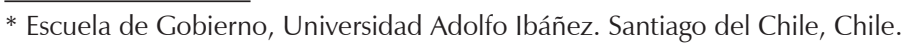




\section{Introducción ${ }^{1}$}

Uno de los temas de nuestro tiempo es la migración. Esto no se relaciona exclusivamente con la cantidad de personas que cambian su residencia en el contexto internacional. Sólo el 3.3\% de los habitantes de algún país son inmigrantes, entendiendo por tales a aquellos que tienen su lugar de nacimiento fuera de las fronteras de su país de residencia ${ }^{2}$. El que el fenómeno migratorio sea un tema de urgencia política y humanitaria se debe, primero, a la organización mundial mediante Estados nacionales que reclaman para sí, y se reconocen mutuamente, un derecho a excluir inmigrantes ${ }^{3}$, y que tienen el poder burocrático para controlar sus fronteras; $y$, segundo, a las grandes desigualdades en oportunidades entre países, que en ocasiones implican condiciones de vida miserables. Según datos de la UNHCR, el año 2015 había más de 65 millones de desplazados forzados. Por cierto, la mayor cantidad buscan refugio dentro de las fronteras del propio país. Pero entre los desplazados forzados la UNHCR cifra en más de 21 millones la cantidad de refugiados internacionales, siendo más de la mitad menores de edad. Según datos del año 2015, esto quiere decir que cada día casi 34.000 personas deben dejar su país de origen huyendo de conflictos armados y persecución. 53\% de los refugiados provienes de 3 países: Somalia (1.1 millones), Afganistán (2.7 millones), y Siria (4.9 millones) ${ }^{4}$. Alcanzando cifras record, la UNHCR proyecta para el año 2017 en 1.19 millones la cantidad de personas necesitadas en búsqueda de reasentamiento, cifra que supera por mucho las oportunidades disponibles en países terceros 5 . Pero la urgencia política y humanitaria va más allá del caso de los refugiados. En buena medida, las causas de la migración refieren a la miseria y falta de oportunidades que suelen acompañar a la pobreza. Vivimos en un mundo caracterizado por grandes desigualdades. En términos del Coeficiente de Gini, que mide la desigualdad de ingresos en una escala entre 0 y 100 (donde 0 implica igualdad perfecta -la distribución es igualitaria- y 100 o 1 máxima desigualdad -uno concentra todo y los otros

\footnotetext{
Este trabajo se inscribe en el Núcleo Milenio Modelos de Crisis (NS130017).

2 Según los datos presentados por las Naciones Unidas para el año 2015, el número de migrantes internacionales, entendiendo por tales aquellos que viven en un país distinto al de su nacimiento, alcanzó 244 millones. Si bien la cifra muestra un aumento de $41 \%$ con respecto a la del año 2000, ella continua siendo relativamente moderada (un 3.3\% de la población global). UNITED NATIONS. International Migration Report 2015. Disponible en: <http:// www.un.org/en/development/desa/population/migration/publications/migrationreport/docs/ MigrationReport2015.pdf>.

3 Esta pretensión sólo se ve cualificada por la Convención de Refugiados de Ginebra, cuyo espíritu y también letra hoy raramente se respeta.

4 Contrariamente a lo que sugiere la prensa, los países que más refugiados cobijan son Jordania, Etiopía, Irán, el Líbano, Paquistán y Turquía.

5 Para un argumento a favor de un amplio derecho a refugio que se construye en base a Kant, compare LOEWE, Daniel. Los náufragos de nuestro tiempo. El caso de los refugiados.
} 
no tienen nada), la desigualdad global es mayor que la desigualdad dentro de cualquier país individual. Según Milanovic ${ }^{6}$, economista del Banco Mundial, esto se debe a que hoy, la mayor diferencia de ingreso entre los individuos se puede atribuir al país del que uno es ciudadano, en contraste con el siglo XIX, en que era atribuible a la posición que cada cual ocupaba dentro de la propia sociedad. En una medida importante, son estas desigualdades las que están a la base de los flujos migratorios. Y nada parece indicar que este sea un escenario temporal: entre las consecuencias del cambio climático se reconoce enormes flujos de desplazados e inmigrantes que serán siendo causa de presión en el orden internacional ${ }^{7}$. Al nivel de la discusión filosófico-política, la actualidad del tema no sólo se debe a la urgencia que el fenómeno impone, sino también y sobre todo, a la tensión que se genera entre el foco estado-céntrico que caracteriza a las teorías de justicia dominantes, y sus propios presupuestos normativos que parecen implicar una ampliación del foco de aplicación de las exigencias de justicia más allá de las fronteras políticas.

En este artículo evaluaré argumentos a favor de la inmigración que recurren en su argumentación a las exigencias normativas de la justicia distributiva. En lo que sigue, al referirme a la justicia me estaré refiriendo a la justicia distributiva. Sostendré que este tipo de estructuras argumentativas tienen un alcance menor y subsidiario, aunque importante ${ }^{8}$. Para esto procederé en cinco pasos. Primero me referiré a la doble función de las fronteras. Luego me refiero a la pregunta acerca del scope de la justicia distributiva. En tercer lugar presentaré concisamente cuatro tipos de argumentos que relacionan la justicia distributiva global con la inmigración. En cuarto lugar examinaré si la migración avanza los fines de la justicia distributiva global. Finalmente me referiré a los límites de este enfoque.

\section{Fronteras y oportunidades}

Es común diferenciar entre pull-factores (los que atraen al inmigrante) y push-factores (los que hacen al emigrante a abandonar su país de origen), y relacionar los primeros con los inmigrantes económicos y los segundos con los refugiados. Pero en muchos casos ambos factores actúan simultáneamente: la atractividad de un destino es una función de la del lugar de origen. Fronteras cumplen una doble función ${ }^{9}$. Por una parte concentran combinaciones de

6 MILANOVIC, Branko. Global inequality. A new approach for the age of globalization.

7 Compare LOEWE, Daniel. Refugiados climáticos: ¿quién debe cargar los costos?

8 En mi opinión, y en contra de esta línea argumentativa, los mejores argumentos a la base de un derecho a la inmigración se articulan en términos de libertad y no de justicia distributiva (LOEWE, Daniel. Inmigración y el derecho de gentes de John Rawls. Argumentos a favor de un derecho a movimiento sin fronteras). En este artículo no desarrollaré esta argumentación.

9 CAVALLERO, Eric. An immigration pressure model of global distributive justice Politics. Por cierto el rol de las fronteras no se agota en lo expuesto. Un análisis del papel de las fronteras en el caso de la migración en LOEWE, Daniel. Fronteras, Liberalismo e Inmigración. 
oportunidades en ciertas circunscripciones. Por otra parte niegan o condicionan el acceso a estas oportunidades a todos aquellos que están afuera. De este modo, ellas distinguen entre todos aquellos que tienen acceso a estas oportunidades y aquellos que no. Las oportunidades pueden ser conceptualizadas de modos diversos. Por ejemplo: libertades, capacidades, bienes primarios, recursos, oportunidades para alcanzar bienestar, bienestar, etc. En lo que sigue utilizaré una definición acotada pero aun indeterminada de oportunidades: oportunidades son bienes materiales relevantes para poder perseguir un plan de vida y que pueden ser objeto de una política distributiva. Teorías igualitarias distributivas suelen distinguir entre las desigualdades en oportunidades dentro de una circunscripción y las desigualdades en oportunidades entre circunscripciones. Al hacerlo, distinguen normativamente entre las oportunidades a las que deben poder acceder los miembros de diferentes circunscripciones. Ya que, como es conocido, en nuestro mundo las oportunidades distribuidas en circunscripciones delimitadas por fronteras varían de un modo extremo, varían también las oportunidades de los individuos para lograr lo que aspiren en la vida. La relación entre igualdad y la migración se puede conceptualizar de modos diferentes. Por una parte, se puede considerar que lo moralmente errado al afirmar la doble función de las fronteras es la segunda premisa: que las fronteras excluyan de las oportunidades que circunscriben a todos aquellos que están afuera. Por otra parte, se puede considerar que lo errado en la afirmación mencionada yace en la primera: las diferencias en oportunidades que se dan en las distintas circunscripciones. En el primer caso, se puede argumentar a favor de la migración en vista de un argumento de libertad. Esta es la posición que yo defiendo (aunque no lo haré acá). Como resulta evidente, este tipo de argumentos no depende de ninguna consideración ni comparación de las oportunidades que las fronteras circunscriben. Para notarlo, basta imaginarse un mundo en el cual todas las circunscripciones concentran en mismo número y tipo de oportunidades. En este mundo, el argumento a favor de la migración basado en la libertad de los individuos para cruzar fronteras seguiría siendo válido -en caso de ser correcto-. En el segundo caso, la aspiración normativa es que los conjuntos de oportunidades circunscritos por las fronteras cumplan con alguna función de igualdad, ya sea absoluta o relativa. En este caso, el argumento a favor de la migración es derivativo de las oportunidades que circunscriben las fronteras. Dicho de otro modo, la migración es legítima sólo en la medida en que ayude a incrementar las oportunidades a las que pueden tener acceso los individuos de modo tal que se cumpla la función igualitaria. En este artículo me enfocaré en argumentos que hacen depender la legitimidad de la migración en consideraciones acerca del tipo y número de oportunidades que circunscriben las fronteras. Esta es la preocupación en el foco de las teorías de justicia distributiva. 


\section{Justicia distributiva y el scope}

La pregunta que una teoría igualitaria no puede obviar es la relativa al scope. Es decir, la pregunta acerca del espacio en que legítimamente se pueden desplegar las demandas igualitarias. ¿Son estas demandas legítimas exclusivamente dentro de las sociedades políticamente organizadas, o lo son también entre sociedades? De un modo general, aquí se oponen las teorías de corte cosmopolita, que articulan un espacio más amplio de aplicación de las demandas igualitarias, y las teorías igualitarias democráticas, que hacen coincidir el espacio con las circunscripciones nacionales. Las teorías de justicia igualitarias suelen ser estado-céntricas. Es decir, sostienen que el foco de la justicia son las sociedades políticamente organizadas.

La respuesta a la pregunta acerca del scope no es independiente de la de la respuesta a la pregunta acerca de la relevancia de la igualdad. El igualitarismo de la suerte articula una respuesta a la pregunta acerca de porque la igualdad material (definida de acuerdo a la distribución de un conjunto definido de bienes -la llamada currency of egalitarian justice-) es importante. Esta respuesta se funda en una intuición sobre el valor igual de los individuos en cuanto agentes. El igualitarismo de la suerte toma la igualdad en la distribución de un cierto conjunto de bienes como punto de partida y considera que cualquier desviación desde esta línea basal igualitaria debe ser justificada. El supuesto de la igualdad como punto de referencia se funda en una interpretación de la igualdad moral de los individuos, mientras que la legitimación de las desviaciones desde esta línea basal se basa en una interpretación del valor moral de su agencia. El resultado de estas interpretaciones parece ser que una distribución desigual de los bienes considerados como relevantes sólo es legítima si ella puede ser retrotraída a la agencia de los individuos. Inversamente: cualquier desviación que se retrotraiga a la pura suerte es ilegítima. G.A. Cohen expresa esta idea general del siguiente modo: "there is injustice in the distribution when the inequality of goods reflects not such things as differences in the arduousness of different people's labors, or people's different preferences and choices with respect to income and leisure, but myriad forms of lucky und unlucky circumstance ${ }^{\prime 10}$. Desde la perspectiva del igualitarismo de la suerte, las diferencias en distribución que se basan en la suerte deben ser neutralizadas por razones de justicia, mientras que las diferencias en distribución que se fundan en elecciones (choice) son perfectamente legitimas.

Hay múltiples modos de entender el contraste entre suerte y agencia como la diferencia entre suerte bruta y opcional, entre elección y circunstancia, etc.-. Correspondientemente hay múltiples modos de trazar la línea entre

\footnotetext{
${ }^{10}$ COHEN, Gerald A. If you are an egalitarian, how come you're so rich?, p. 130.
} 
ellas ${ }^{11}$. Estas son discusiones relevantes que se relacionan con la pregunta acerca de qué es una distribución justa ${ }^{12}$. Yo no entraré en esta discusión. Para los objetivos de este artículo basta con afirmar que (i) es moralmente relevante la diferencia entre elecciones y circunstancias no escogidas (esto es, el núcleo del igualitarismo de la suerte), cualquiera sea la línea trazada entre ellos, y (ii) que las circunstancias relevantes son, al menos, de tipo social y natural. En este sentido, es suficientemente persuasivo afirmar que el contexto de socialización es tanto un asunto de circunstancias no escogidas como lo es la dotación natural física y cognitiva (en oposición a elección), y que contamos con razones de justicia para neutralizar (ya sea ex ante o ex post) los efectos de las circunstancias no escogidas en la vida ${ }^{13}$.

En sentido estricto, la teoría de justicia de Rawls no es una teoría que se base en el igualitarismo de la suerte. Aunque la versión de 1971 admite esa lectura, la teoría de Rawls puede ser mejor entendida como fundada en un tipo de igualdad democrática ${ }^{14}$. La igualdad democrática expresa un ideal político fundado en una idea de democracia y no en un ideal moral. De acuerdo a Rawls, la sociedad es un sistema de cooperación y los principios de justicia gobiernan la distribución de sus beneficios y cargas. Esos principios deben ser los que individuos libres e iguales razonablemente pueden aceptar mutuamente. Esta concepción implica igualdad de algún conjunto de bienes, porque esta igualdad es parte de los principios que cada cual tiene buenas razones para aceptar. La regulación de las desigualdades se sigue de un principio

${ }^{11}$ Compare ARNESON, Richard. Equality and equal opportunity of welfare; ARNESON, Richard. Against Rawlsian equality of opportunity; COHEN, Gerald A. On the currency of egalitarian justice; DWORKIN, Ronald. Equality, luck, and hierarchy; -una discusión en TAN, Kok-Chor. Justice, institutions, and luck, p. 88.

${ }^{12}$ A modo de ejemplo: inuestras preferencias rechazadas pero no modificables deben ser consideradas como elecciones o como circunstancias, y correspondientemente como preferencias costosas o como elementos a ser compensados? ¿Y qué sucede con las disposiciones productivas: son resultado de nuestra agencia o de circunstancias afortunadas en la vida como sostiene Rawls?

${ }^{13}$ La preocupación por la (des)igualdad en la distribución no lo es exclusivamente del igualitarismo de la suerte. Hay también otras respuestas acerca de por qué la igualdad es importante. El contraste al igualitarismo de la suerte está dado por el denominado igualitarismo "social", "relacional" o "democrático" (compare SHEFFLER, Samuel. What is egalitarianism?; SHEFFLER, Samuel. Equality and Tradition; ANDERSON, Elizabeth. What is the point of equality; ANDERSON, Elizabeth. How should egalitarians cope with market risks?; MILLER, David. Justice and inequality). Desde estas perspectivas, la justicia de cualquier estado de cosas debe ser juzgada desde la perspectiva de la igualdad de las relaciones socio-políticas relevantes (para esta definición y una discusión de esta perspectiva, compare SEGALL, Shlomi. Equality and opportunity, p. 16). Evidentemente, la igualdad sigue siendo central y tiene consecuencias importantes. Pero la igualdad es importante porque las relaciones socio-políticas entre los miembros de un orden socio-político son importantes, y no porque las personas en cuanto tal estén en una relación de igualdad moral fundada en la distinción entre suerte y agencia o entre circunstancias no escogidas y elecciones.

14 RAWLS, John. Justice as fairness. 
de reciprocidad o fairness que gobierna las relaciones entre los miembros de la sociedad. Así, al referir a la justificación del principio de la diferencia afirma Rawls que "democratic equality properly understood requires something like the difference principle"15. En contraste al igualitarismo de la suerte, la (des) igualdad no tiene relevancia moral última. Su relevancia se sigue de la igualdad democrática de los miembros de la sociedad. Por cierto, para esta concepción de justicia la distinción entre decisiones y circunstancias no escogidas es central. Pero es derivativa de la concepción de igualdad democrática.

¿Qué se sigue para la pregunta del scope? El igualitarismo de la suerte no tiene una restricción del scope en su arquitectura. La respuesta que articula a la pregunta de por qué la igualdad es importante, se basa en una distinción entre agencia y el valor moral de los individuos. Y esta respuesta es válida independientemente del lado de la frontera en que se encuentren los individuos. Si las oportunidades circunscritas por las fronteras desaventajan de un modo relevante a los individuos, desde la perspectiva del igualitarismo de la suerte tenemos razones para sostener que esta desventaja es inmerecida y que, por tanto, debe ser neutralizada mediante principios de justicia igualitaria. Así considerado, el igualitarismo de la suerte es cosmopolita (lo que no significa que autores que articulan sus teorías asumiendo la premisa del igualitarismo de la suerte defiendan posiciones cosmopolitas, lo que, obviamente no es el caso). Tomar en serio la premisa normativa a su base (la distinción entre agencia y suerte) permite extender las exigencias de la justicia igualitaria. Cualquier restricción del scope sólo puede deberse a la consideración de otros valores a los que se otorgue primacía por sobre el de la igualdad. Por otra parte, el igualitarismo democrático reduce el scope al conjunto de todos aquellos que están en la relación recíproca. En este caso, la neutralización de la (mala) fortuna no aplica a los que están más allá de las fronteras. Ciertamente puede haber otras razones para atender a la condición de aquellos que se encuentran fuera de las fronteras. Pero estas no son razones de justicia igualitaria en sentido estricto. Ellas pueden ser razones de humanidad o razones morales que atiendan a niveles absolutos (y no relativos).

\section{Justicia distributiva global e inmigración}

En el caso de la migración, la referencia a la justicia distributiva pone en cuestión el primer papel de las fronteras: circunscribir conjuntos de oportunidades. El imperativo moral y político es que las combinaciones de oportunidades que circunscriben los diferentes países deben ser consideradas desde alguna perspectiva normativa. Desde esta perspectiva, lo moralmente problemático es que los miembros de una sociedad dispongan de oportunidades

${ }^{15}$ Ibidem, p. 49; RAWLS, John. A theory of justice, p. 65-66. 
reducidas o de poco valor. La institucionalización de la premisa cosmopolita, de acuerdo a la cual nadie debe ser sistemáticamente desaventajado por contingencias como la ciudadanía o la nación de origen, implica, antes que nada, medidas de justicia distributiva de alcance global que aseguren una cierta cantidad y calidad en las oportunidades en las diferentes sociedades. El derecho a inmigrar es subsidiario. Es decir, la inmigración sólo es legítima en tanto avance el fin de la justicia distributiva global: que los individuos tengan acceso a una cierta pluralidad y calidad de opciones.

Esta posición se basa en dos premisas: (i) hay obligaciones de justicia distributiva global; y (ii) la inmigración es un mecanismo que puede avanzar los fines de la justicia distributiva global ${ }^{16}$. A continuación examinare los argumentos en los cuales se sustentan estas premisas.

\subsection{Liberalismo igualitario}

Los argumentos a favor de una obligación de justicia global más populares en la actualidad, siguen la estructura de las teorías igualitarias. Las obligaciones de justicia distributiva global se basarían en que la arbitrariedad de nacer en una sociedad particular no puede implicar diferencias en oportunidades y por consiguiente en expectativas de vida tan grandes como en nuestro mundo. Una vez que, a un nivel fundamental, aceptamos la premisa de la igualdad de los seres humanos, todos deberían tener acceso a un conjunto similar o al menos decente de oportunidades. Los principios de justicia distributiva global están llamados a mitigar -directamente o en forma subsidiaria- estas consecuencias del azar del nacimiento.

Este tipo de argumentos se han desarrollado extensiva e intensivamente por referencia a la teoría de justicia de John Rawls. Como examinamos, Rawls reduce la aplicación de principios de justicia a sociedades políticamente organizadas ${ }^{17}$. Utilizando sus conceptos, la suya sería una teoría de justicia social, es decir, una teoría acerca de la justicia de las instituciones más importantes que componen la estructura básica de la sociedad. Sin embargo, como autores cosmopolitas han argumentado, esta restricción del foco de la justicia no se condeciría con la premisa igualitaria en base a la cual Rawls construye su teoría de justicia ${ }^{18}$. Esta lectura no se puede deducir del igualitarismo democrático que Rawls afirma en Liberalismo Político, sino más bien definición de la clase de los sujetos de justicia por referencia a la posesión de los dos poderes morales (poder desarrollar una concepción del bien, y tener un sentido de justicia), y a la distinción entre fortuna y responsabilidad por el propio plan de vida.

\footnotetext{
${ }^{16}$ Compare WILCOX, Shelley. The open borders debate on immigration, p. 817.

17 RAWLS, A theory..., op. cit.; RAWLS, John. Political liberalism.

${ }^{18}$ BARRY, Brian. The liberal theory of justice; BARRY, Brian. Theories of justice; BEITZ, Charles. Political theory and international relations; POGGE, Thomas. Realizing Rawls.
} 
La intuición igualitaria a la base de la teoría de Rawls, es que hay factores por los que no podemos ser considerados como responsables. Nadie merece su lugar económico o social inicial, o sus talentos naturales. Rawls incluye dentro de estos factores incluso la posesión de virtudes productivas (responsabilidad, laboriosidad, etc.) que influyen profundamente en lo que podemos alcanzar en la vida ${ }^{19}$. Y si no merecemos estos factores, entonces tampoco podemos razonablemente cargar con las desventajas que producen. Sin cualificaciones, en un plano de teoría ideal, la premisa igualitaria torna difícil considerar a las fronteras políticas, hechos contingentes y accidentes de la historia, como moralmente relevantes ${ }^{20}$. Principios de justicia están llamados e corregir, o neutralizar estos resultados. Como muchos autores han señalado (y Rawls ha rechazado $\left.{ }^{21}\right)^{22}$, en esta ecuación habría que incluir otros factores arbitrarios por los que nadie puede ser considerado como responsable y que influencian de un modo casi determinante lo que podemos lograr en la vida. Dentro de estos factores la ciudadanía y el origen nacional ocupan un lugar central. Aunque en sentido estricto nadie los merece, van a la par de expectativas de vida radicalmente distintas. En esta línea argumentativa afirma Joseph Carens que el estatus de la ciudadanía en las democracias liberales occidentales es el equivalente moderno del privilegio feudal: un estatus heredado que amplia nuestras oportunidades en la vida ${ }^{23}$. Si esto es así, entonces los principios de justicia debiesen neutralizar estos factores. Una teoría liberal igualitaria cosmopolita debe hacerse cargo tanto del problema de la desigualdad económica mundial, como también de la pretensión de universalidad de determinados valores. Un modo de hacerlo, sería mediante el reconocimiento de obligaciones de justicia distributiva global (que Rawls expresamente rechaza ${ }^{24}$ ). Otro modo, mediante el reconocimiento de un derecho a movilidad sin fronteras ${ }^{25}$. Mediante este derecho los individuos se asegurarían la posibilidad de poder acceder a otras sociedades para llevar a cabo su plan de vida. La movilidad libre parece estar enquistada así en el núcleo moral del liberalismo igualitario ${ }^{26}$. Así, se podría avanzar en la neutralización de los efectos devastadores de la lotería natural.

\footnotetext{
${ }^{19}$ RAWLS, A theory..., op. cit., p. 104.

${ }^{20}$ En otro sitio he discutido el papel de las fronteras con respecto a la migración (LOEWE, Daniel. Fronteras, Liberalismo..., op. cit.).

${ }^{21}$ RAWLS, John. The law of peoples with "The idea of public reason revisited".

${ }^{22}$ En otro sitio he discutido The Law of Peoples de Rawls (LOEWE, Daniel. ¿Cuán liberal es la teoría de las relaciones internacionales de Rawls?).

${ }^{23}$ CARENS, Joseph. Aliens and citizens. The case of open borders.

${ }^{24}$ RAWLS, The law of..., op. cit.

${ }^{25}$ Compare LOEWE, Daniel. Inmigración y el derecho..., op. cit.

${ }^{26}$ Para defensas de políticas de open borders compare, entre muchas otras, VELASCO, Juan Carlos. El azar de las fronteras; LOEWE, Daniel. Inmigración y el derecho..., op. cit.; SHACHAR, Ayelet. The Birthright Lottery. Citizenship and Global Inequality.
} 


\subsection{Deber de compensación}

Otro tipo de argumento relaciona la justicia distributiva global con obligaciones de compensación. Como ha señalado Thomas Pogge, la organización del contexto internacional produce externalidades que influyen de un modo importante en el desarrollo económico de las naciones. Las causas que explican el fracaso de algunas naciones en la determinación de medidas políticas y económicas acertadas no se relaciona exclusivamente con factores internos como corrupción, incapacidad, cultura política, etc. Si bien estos factores juegan un papel central, para explicar la pobreza de algunas naciones hay que atender también a las consecuencias de otros factores con un carácter global en las políticas nacionales, como por ejemplo las prácticas comerciales, los patrones de consumo de las naciones ricas, el derecho internacional, leyes de patentes, etc $^{27}$. De este modo, de estas consideraciones acerca de la responsabilidad por los efectos de un sistema internacional que favorece a algunos más que a otros, se desprenden obligaciones de justicia distributiva global.

Directamente relacionado con el argumento anterior, habría responsabilidades compensatorias que se retrotraen a la historia. Como Benhabib argumenta ${ }^{28}$, las circunstancias en las cuales los pueblos toman sus decisiones están influenciadas por factores históricos como, por ejemplo, los procesos de colonización y sus consecuencias. Estas son informaciones relevantes para determinar qué es lo que se debe a determinadas naciones $-y$ también, como señala Höffe, para determinar quién es el deudor ${ }^{29}$.

\subsection{Copropiedad de la tierra}

Un tercer tipo de argumento de corte iusnaturalista recurre a las teorías de la posesión común de la tierra y, desde esta, deriva obligaciones de justicia distributiva global. En la actualidad Mathias Risse ha defendido en On Global Justice vigorosamente esta idea ${ }^{30}$. En un cierto sentido, el ciudadano del mundo, al que se suele apelar en las teorías cosmopolitas y de justicia global $^{31}$, se caracteriza aquí como un copropietario de los recursos originales del planeta. De la posesión común de la tierra se seguirían exigencias de justicia global que aseguren la satisfacción de necesidades básicas. La legitimidad de las convenciones de propiedad vigentes depende del cumplimiento de esta restricción. Así, estas convenciones deben (i) garantizar a los copropietarios la oportunidad para utilizar los recursos originales de la tierra para satisfacer

\footnotetext{
${ }^{27}$ POGGE, Thomas. World poverty and human rights.

${ }^{28}$ BENHABIB, Seyla. The Rights of Others, p. 100.

${ }^{29}$ HÖFFE, Otfried. Demokratie in Zeitalter der Globalisierung.

${ }^{30}$ Una discusión en LOEWE, Daniel. Humanity's collective ownership of the earth and immigration.

${ }^{31}$ LOEWE, Daniel. Cinco dimensiones del cosmopolitismo.
} 
necesidades básicas, (ii) o garantizar la oportunidad para satisfacer necesidades básicas de otros modos. Se trata de una exigencia de justicia. En el caso de la inmigración hay que diferenciar dos situaciones. En la primero, las necesidades básicas de algunos copropietarios no son satisfechas. En la segunda sí lo son. La teoría de justicia global de Risse aspira a que las necesidades básicas de los copropietarios sean satisfechas allí donde se encuentren: "It is part of my proposal that the response required to scenarios in which individuals cannot satisfy basic needs is to make sure they can do so where they live, rather that permit immigration for that reason" ${ }^{\prime 32}$. Por lo tanto, la demanda de justicia efectiva en casos de no satisfacción de necesidades básicas no es, primeramente, una restricción a la potestad de los Estados para excluir inmigrantes, que aspiren a ingresar para satisfacer sus necesidades básicas mediante los recursos originales comunes, sino que es una demanda para que se garantice la oportunidad de satisfacer necesidades básicas en el lugar en que se encuentren los copropietarios. Sin embargo, es fácil notar que si esto no es posible, puede surgir una demanda legítima expresada en términos de justicia para que no se excluya a los inmigrantes con necesidades básicas insatisfechas. Este puede ser el caso, por ejemplo, de los habitantes de las islas destinadas a desaparecer producto del aumento del nivel de los mares que trae consigo el calentamiento global -un caso que Risse ha tratado-. Así, de acuerdo a esta teoría: "the inhabitants of Kiribati, like all human beings, are co-owners of the earth and as such have claims to relocation in the event that their existence becomes impossible where they presently live and regardless of who was responsible for creating this situation" ${ }^{\prime 3}$. Pero también podría ser el caso de copropietarios con necesidades básicas insatisfechas, pero que no pueden ser satisfechas en sus países de origen, tales como los refugiados. En este tipo de casos acotados, la teoría sí podría tener un rendimiento positivo con respecto a la inmigración.

Ahora bien, asumiendo que las necesidades básicas de todos los copropietarios son satisfechas allí donde se encuentran iqué se sigue del enfoque de la propiedad común en el caso de la inmigración? Risse responde a esta pregunta mediante una teoría de uso proporcional, según la cual hay que examinar si los Estados están subutilizando o sobreutilizando los recursos originales en comparación con los otros Estados. La idea es la siguiente: para cada Estado hay una medida del valor de los recursos originales de propiedad colectiva dentro de su territorio. Este valor incluye las condiciones biofísicas que determinan la utilidad de esta región para propósitos humanos (tales como clima, vegetación, ubicación, topografía, etc.). Al dividir este valor por

\footnotetext{
${ }^{32}$ RISSE, Mathias. On Global Justice, p. 153.

33 IDEM. The Right to Relocation: Disappearing Island Nations and Common Ownership of the earth, p. 282. Una consideración hacia los refugiados medioambientales que no se basa en la propiedad común, en LOEWE, Refugiados climáticos..., op. cit.
} 
la población de ese territorio se obtiene la medida de uso de este territorio expresada como la tasa de uso per-capita de los recursos de propiedad común en ese territorio. El valor incluye recursos que no circulan en el mercado, tales como minerales o recursos no explotados. Para posibilitar la comparación de la tasa de uso per-capita entre diferentes Estados, Risse recurre a una medida unidimensional como un precio de mercado mundial. La tasa de uso per-capita de cada Estado, expresada mediante una unidimensional medida, se compara así con la tasa de uso per-capita promedio a través de los Estados. Si el valor es mayor que el promedio a través de los Estados, entonces el territorio del Estado está relativamente siendo subutilizado. Si el valor es menor, entonces está relativamente siendo sobreutilizado. Otro modo de expresarlo, es afirmar que cuando un Estado subutiliza sus recursos, entonces la persona promedio en ese Estado usa un paquete de recursos de mayor valor que la persona promedio en el país promedio, y cuando un Estado está sobreutilizando sus recursos, entonces la persona promedio usa un paquete de recursos de menor valor que la persona promedio en el país promedio. Según la teoría del uso proporcional, si el valor "is above average, co-owners elsewhere have a pro tanto claim to immigration, in the sense that underusing countries cannot reasonably expect other to comply with immigration policies until such claims are satisfied"34. Esto vale hasta que la tasa de uso promedio de los estados se asemeje. De acuerdo a Risse, Estados que subutilizan pueden pagar en vez de aceptar la entrada de extranjeros. Sin embargo, si los inmigrantes en tanto copropietarios de los recursos comunes prefieren entrar, ellos no puede descargar su obligación pagando.

Sin embargo, cuando la demanda de justicia para la satisfacción de necesidades básicas que se sigue de la propiedad común mediante recursos originales ya ha sido satisfecha dentro de los Estados singulares, las obligaciones que se siguen de la teoría del uso proporcional serían de otro tipo. Se trataría de demandas de conducta razonable. Según Risse, obligaciones de justicia referirían a exigencias normativas especialmente importantes, y cuyo cumplimiento puede ser exigido. Este sería el caso de las que refieren a la satisfacción de necesidades básicas. Si bien es razonable que los Estados que subutilizan no establezcan políticas que rechacen a los inmigrantes potenciales provenientes de Estados que sobreutilizan, esto no sería exigible como un asunto de justicia. Risse suele ilustrar este punto con un experimento mental ${ }^{35}$ : imaginemos que la población de EEUU se reduce a dos o tres individuos que mediante un sistema tecnológico avanzado pueden controlar las fronteras. Evidentemente se trataría de un caso de subutilización de recursos naturales. En este caso,

\footnotetext{
${ }_{34}$ RISSE, On global..., op. cit., p. 154.

35 Ibidem, cap. 8; RISSE, Mathias. Response to Arneson, de Bres, and Stilz; una ilustración ligeramente diferente en RISSE, Mathias. On the morality of Immigration.
} 
habría una demanda de conducta razonable hacia ellos para que dejen entrar inmigrantes. Sin embargo, ya que no se trata de una demanda de justicia (porque las necesidades básicas de los copropietarios ya han sido satisfechas), no se puede exigir que los dejen entrar. De igual modo, no se puede exigir a los inmigrantes potenciales que no intenten acceder al territorio. Esto sería una demanda de conducta irrazonable. Así, estos pueden intentar ingresar, y los primeros intentar evitarlo. Según Risse, mientras no se utilice violencia y por tanto no se violen los derechos de los involucrados en este proceso, no se ha violado ninguna demanda de justicia. Lo que se ha violado, por parte de los Estados que subutilizan y tratan de impedir la inmigración, es una demanda de conducta razonable ${ }^{36}$.

\subsection{Utilitarismo y la moralidad de la inmigración}

Un cuarto argumento se construye en base a la doctrina moral y política utilitarista. Como es conocido, esta propone un único principio de moralidad para guiar nuestros actos o la determinación de las reglas e instituciones sociales: la mayor felicidad del mayor número. Una posibilidad es que utilitarismo sostenido sea hedonista. La versión más popular de esta concepción caracteriza la felicidad como "disfrute del placer, resguardo de dolores" ${ }^{\prime 37}$. Aquí se identifica la felicidad con estados mentales caracterizados por la presencia de placer y la ausencia de dolor. Otra posibilidad es definir la felicidad como satisfacción de preferencias. De acuerdo a este entendimiento, la felicidad o bienestar subjetivo de un individuo es el resultado de la satisfacción de sus preferencias o deseos. Este uso es ampliamente extendido en las ciencias económicas. La felicidad se identifica con estados del mundo en que estas preferencias son satisfechas $^{38}$. Una variación de la posibilidad anterior es identificar la felicidad con la satisfacción de intereses fundamentales que podemos razonablemente suponer que todos los individuos comparten ${ }^{39}$. En cualquiera de estos casos, bien entendida, la igual consideración moral de los individuos implica que el principio se debe aplicar a la humanidad como un todo. La función de utilidad debe ser universal (y no nacional). El universalismo yace a la base del utilitarismo. Ciertamente va incluso más allá de la especie humana, si consideramos que lo que está a la base del principio de la felicidad es la capacidad de sintiencia. Desde esta perspectiva, una política de fronteras abiertas sería un instrumento institucional que maximizaría la felicidad total. Citando a King: "Es evidente que el número de gente que gana con esta política [de inmigración irrestricta] excede a todos los que pierden con ella, y si bien los ganadores incluyen

\footnotetext{
${ }^{36}$ RISSE, Response to..., op. cit., p. 521.

${ }^{37}$ BENTHAM, Jeremy. The Principles of Moral and Legislation, cap. 7.

${ }^{38}$ GRIFFIN, James. Well-Being: Its Meaning, Measurement and Moral Importance; HARSANYI, John. Essays on Ethics, Social Behavior, and Scientific Explanation.

${ }^{39}$ HARE, Richard M. Moral Thinking; SINGER, Peter. The life you can save.
} 
algunos de los ricos del mundo, los grupos más pobres también ganan"40. Y recurriendo a los supuestos acerca de la teoría económica neoclásica Hayter sostiene que la política óptima de inmigración sería no tener ninguna ${ }^{41}$. Esta es una argumentación corriente de economistas liberales a favor de la movilidad libre $^{42}$.

Una variación del argumento utilitarista se encuentra en la afirmación del carácter moral de las obligaciones distributivas de alcance global. Este argumento ha sido popularizado por Peter Singer en su cruzada en favor de motivar y canalizar donaciones particulares con el fin de acabar con la pobreza global. La obligación de mitigar la pobreza no es (en todos los casos) una obligación estrictamente de justicia y responsabilidad. Tampoco es simplemente una obligación supererogatoria o de caridad. De lo que se trata, es de una obligación moral de carácter humanitario. De acuerdo a Peter Singer, si podemos evitar que se produzca algo moralmente malo sin sacrificar nada comparable, estamos obligados a hacerlo ${ }^{43}$. Por ejemplo, si podemos salvar a un niño desconocido ahogándose en una pileta (incluso sin ser responsables de que el niño se encuentre en esa situación) al costo de estropear nuestros zapatos, estamos moralmente obligados a hacerlo. Por paridad de razonamiento, si podemos aliviar la pobreza (la pobreza que mata, como señala Singer) mediante donaciones, estamos moralmente obligados a hacerlo, independientemente de la ubicación geográfica o de la nacionalidad de los afectados ${ }^{44}$.

Es difícil descartar la totalidad de estos argumentos a favor de la justicia global. Aunque no estemos de acuerdo con las consecuencias institucionales que el reconocimiento de su fuerza vinculante traería consigo, es poco convincente argumentar que este tipo de obligaciones no existe y que por tanto no deben tener ninguna consecuencia institucional. Si no nos convence el primer argumento que recurre a la igualdad y por tanto a la justicia como neutralizadora de los efectos de la fortuna (porque, por ejemplo, no consideramos que todas las diferencias que se retrotraen a la fortuna son moralmente relevantes), todavía sigue siendo válido el segundo que se basa en

${ }^{40}$ KING, Timothy. Immigration from developing countries: some philosophical issues, p. 527 (Trad. D.L.).

${ }^{41}$ HAYTER, Teresa. Open borders: the case against immigration controls.

42 LEGRAIN, Philip. Immigrants: your country needs them. Compare también el artículo de The Economist: <http://www.economist.com/node/21526893>.

${ }^{43}$ SINGER, Peter. Famine, Affluence, and Morality; IDEM. The life..., op. cit.

${ }^{44}$ Ibidem. La diferencia central entre Singer (Famine, Affluence..., op. cit.) y Singer (The life..., op. cit.), es que en el primero la obligación se desprende de un cálculo de utilidad objetivo, mientras que en el segundo Singer deja en manos de los individuos la decisión acerca de que sacrificio sería comparable. Evidentemente, este movimiento argumentativo tiene como objetivo motivar a los individuos a donar. 
consideraciones sobre responsabilidad y compensación. Y aunque no estemos de acuerdo ni con el primero ni con el segundo, todavía queda el tercer tipo que se basa en la posesión común de la tierra como idea regulativa de la que se desprende un derecho a subsistencia de los copropietarios. Y si no nos convence esta teoría, que dicho sea, tiene dificultades importantes, todavía quedan las consideraciones de responsabilidad moral del cuarto argumento que se basan en cálculos utilitaristas o en ponderaciones valorativas.

\section{4. ¿Avanza la migración los fines de la justicia distributiva?}

Examinemos ahora la segunda premisa, de acuerdo a la cual la inmigración es un mecanismo efectivo para alcanzar los fines de la justicia distributiva global. Se han desarrollado variadas estrategias para dar cuenta de nuestras obligaciones de justicia global. Entre otras, podemos mencionar la implementación de un sistema impositivo global sobre transferencias, un sistema global de dividendos sobre recursos, transferencias directas de ayuda tanto material como tecnológica, promoción de ciertos derechos humanos y capacidades (empoderar a las mujeres, etc.). La migración sería un mecanismo más. A favor de la efectividad de la migración por sobre la de la ayuda material directa parecen hablar una serie de argumentos ${ }^{45}$.

Demasiado a menudo, la ayuda material o buena parte de esta se pierde en administración ineficiente, proyectos más diseñados, y elites corruptas ${ }^{46}$. Por su parte, las oportunidades de la migración sólo están disponibles para todos aquellos que toman ventaja de ésta. Además, las remesas que la suelen acompañar implican asistencia directa y controlada. Y sus efectos positivos no sólo alcanzan a los receptores directos, sino que en la economía nacional como un todo ${ }^{47}$.

A diferencia de la justicia distributiva global, ya que se trata de una respuesta colectiva a la pobreza mundial, la inmigración no implica una carga en algunos individuos (los más ricos), sino que distribuye esta carga entre las diferentes sociedades. Si además consideramos que la inmigración suele ser un motor de desarrollo económico, este modo de hacer frente a la pobreza global iría acompañado de réditos positivos totales en las sociedades receptoras, a diferencia de las transferencias económicas que repercutirían como costos para las sociedades contribuyentes. Además, esta posición sería más consistente con el liberalismo, por cuanto sería la expansión de la libertad

\footnotetext{
${ }^{45}$ WHELAN, Frederick. Citizenship and freedom of movement: an open admission policy?

${ }^{46}$ EASTERLY, William. The White Man's Burden: Why the West's Efforts to Aid the Rest Have Done So Much III and So Little Good.

47 RAPOPORT, Hillel, DOCQUIER, Frédéric. The economics of migrants? Remittances; ADAMS, Richard, PAGE, John. Do international migration and remittances reduce poverty in developing countries?, p. 1660.
} 
(mediante el reconocimiento de la libertad de inmigrar) y el reconocimiento de la responsabilidad individual -de los inmigrantes- lo que llevaría a disminuir las desigualdades materiales, y no algún tipo de asistencialismo.

Por otra parte se han desarrollado argumentos convincentes contra la migración como mecanismo para disminuir la pobreza en el mundo ${ }^{48}$. Como Pogge ha señalado, la migración no resolverá el problema de la pobreza global. Esto se debería a que el número de personas necesitadas en el mundo, que él estima en al menos mil trescientos millones, excede bastamente el número de inmigrantes que los países afluentes podrían admitir ${ }^{49}$. Además, la migración a países ricos no es algo que beneficie directamente a los peor situados en las sociedades de origen. Esto lo podemos considerar desde dos perspectivas. Desde la perspectiva de la nación receptora, desde el momento en que se debe limitar la cantidad de inmigrantes que ingresa, y en tanto la selección no sea completamente aleatoria, se tenderá a privilegiar a todos aquellos individuos que pueden resultar beneficiosos para el funcionamiento de la economía nacional. Pero todos aquellos que pueden ofrecer las habilidades solicitadas no son usualmente los peor situados en las sociedades de origen ${ }^{50}$. Por su parte, desde la perspectiva de la nación de origen, los más dispuestos y capaces para migrar no son usualmente los más pobres. El proceso de migración requiere de una serie de precondiciones, de tipo económico, cultural, etc. pero también se ve facilitada por la existencia de redes familiares y comunales. Pero los peor situados en la sociedad de origen usualmente no tienen acceso a los recursos que exige la migración. Directamente relacionado con lo anterior, los que debido a los procesos migratorios abandonarán la sociedad en busca de mejor fortuna son aquellos que tienen algunas de las capacidades y habilidades más necesitadas en los países de origen (fuga de cerebros), lo que perjudica a la sociedad de origen disminuyendo las probabilidades de que supere la pobreza $^{51}$.

\section{Límites de la justicia distributiva en el caso de la inmigración}

Si la motivación es la superación de la pobreza global, es decir, algún tipo de nivelación de las oportunidades disponibles en las distintas sociedades, la perspectiva de la justicia distributiva global tiene mucho a su favor. Sin embargo,

\footnotetext{
${ }^{48}$ Compare BROCK, Gillian. Global justice: a cosmopolitan account.

${ }^{49}$ POGGE, Thomas. Migration and poverty.

${ }^{50}$ Ibidem.

${ }^{51}$ BROCK, op. cit. Este último argumento tiene cierta debilidad. Si la óptica normativa es individual, es una razón débil frente al emigrante potencial capacitado. Además, el que los más necesitados para superar la pobreza emigren no implica que ellos tengan alguna oportunidad de aplicar sus capacidades en la nación de origen. Pero si el foco del interés es exclusivamente la pobreza mundial y el criterio normativo prevalente es su eliminación, entonces este argumento adquiere cierta fuerza.
} 
hay que considerar que desde esta perspectiva se debe estar dispuesto a aceptar inmigrantes toda vez que no sea posible mejorar su posición mediante otros mecanismos de justicia distributiva global, como transferencias de recursos y tecnología, ayuda al desarrollo, etc. El caso más evidente es el de los refugiados. Pero, evidentemente, esta obligación se extiende también al caso de los inmigrantes económicos o a algunos de ellos que se encuentran en situaciones que menoscavan su vida. Sin embargo, podemos ir más allá. En muchos casos los mecanismo de la justicia distributiva global, aun si son exitosos, requieren de mucho tiempo para ser efectivos. Por otro lado, la urgencia de la necesidad es imperiosa. Lo que se pierde hoy debido a la pobreza (oportunidades de educación, salud, esperanza de vida, etc.), no se obtiene en el futuro cuando los mecanismos de la justicia distributiva global sean efectivos. En ese futuro promisorio los necesitados de hoy estarán muertos. Por lo tanto, aunque no sostengamos un derecho a la inmigración o a movilidad libre, si lo que guía nuestro actuar son los individuos y sus derechos (y no la maximización de una agregación de derechos satisfechos) mucho parece hablar a favor de no imposibilitar la migración hoy de todos aquellos que lo requieran para escapar de situaciones de pobreza.

En muchos casos, la defensa de la justicia distributiva global deja una sensación ambivalente. Este es sobre todo, aunque no exclusivamente, el caso de autores que argumentan a su favor desde posiciones que podemos asociar con el así llamado liberalismo nacional ${ }^{52}$. Por una parte se reconoce la legitimidad de algún tipo de demanda de justicia distributiva global. Y por lo mismo se reconoce que la pobreza extrema de algunas geografías puede ser una razón para recibir inmigrantes con ese origen en sociedades liberales. Este es el caso, por ejemplo, de David Miller en su crítica al derecho a movilidad sin fronteras ${ }^{53}$. Por otra parte, la conclusión que se obtiene de las premisas mencionadas es que, para legítimamente poder negar el ingreso a todos aquellos individuos necesitados, tenemos que cumplir nuestras obligaciones de justicia distributiva global. El control de las fronteras se compra con la billetera. Por una parte, esta posición reconoce y quiere hacer efectivas las obligaciones de justicia global. Por otra parte, estas teorías despiertan la impresión de que en vistas a la amenaza que representa la inmigración para las culturas nacionales, el mal menor es la justicia distributiva global.

Pero aun cuando los mecanismos de la justicia distributiva global sean efectivos, y de este modo los conjuntos de oportunidades que circunscriben las sociedades políticamente organizadas sean igualadas, es razonable suponer que estos todavía variaran significativamente en cuanto a su cantidad y calidad.

${ }^{52}$ KYMLICKA, Will. Liberalism, community and culture; IDEM. Multicultural citizenship; MILLER, David. On Nationality; TAMIR, Yael. Liberal nationalism.

${ }^{53}$ MILLER, David. Justice and..., op. cit.; IDEM. National responsibility and global justice. 
Si la teoría de justicia distributiva es suficientarista, y por tanto refiere sólo un mínimo vinculante para todas las sociedades, esto no es problemático. Pero si la función igualitaria de la teoría de justicia distributiva global que se defiende es más exigente, entonces la consecuencia mencionada puede ser problemática. Un derecho a movilidad libre podría disminuir aún más la desigualdad en tanto pondría, mediante la inmigración, a disposición de los individuos las oportunidades disponibles en otras sociedades ${ }^{54}$.

\section{Bibliografía}

ADAMS, Richard; PAGE, John. Do international migration and remittances reduce poverty in developing countries? World Development, v. 33, n. 10, 2005, p. 1645-1669.

ANDERSON, Elizabeth. How should egalitarians cope with market risks? Theoretical Inquiries in Law, v. 9, 2008, p. 239-270.

ANDERSON, Elizabeth. What is the point of equality. Ethics, v. 109, n. 2, 1999, p. 287-337.

ARNESON, Richard. Against Rawlsian equality of opportunity. Philos Stud, v. 93, 1999, p. 77-122.

ARNESON, Richard. Equality and equal opportunity of welfare. Philos Stud, v. 56, 1989, p. 77-93.

BARRY, Brian. The liberal theory of justice. Oxford: Oxford University Press, 1973.

BARRY, Brian. Theories of justice. California: University of California Press, 1989.

BARRY, Brian; GOODING, Robert (eds.). Free movement: ethical issues in the transnational migration of people and money. Pennsylvania: Penn State University Press, 1992.

BEITZ, Charles. Political theory and international relations. Princeton: Princeton University Press, 1979.

BENHABIB, Seyla. The Rights of Others. Cambridge University Press, 2004.

BENTHAM, Jeremy. The Principles of Moral and Legislation. New York: Hafner, 1948.

BROCK, Gillian. Global justice: a cosmopolitan account. Oxford: Oxford University Press, 2009.

CANEY, Simon. Cosmopolitan justice and equalizing opportunities. Metaphilosophy, v. 32, 2001, p. 113-134.

CANEY, Simon. International distributive justice. Political Studies, v. 49, n. 5, 2001, p. 974-997.

CARENS, Joseph. Aliens and citizens. The case of open borders. Review of Politics, v. 49 , n. 2,1987 , p. 251-273.

${ }^{54}$ LOEWE, Inmigración y el..., op. cit.; CANEY, Simon. Cosmopolitan justice and equalizing opportunities; IDEM. International distributive justice. 
CARENS, Joseph. Migration and morality: a liberal egalitarian perspective. In BARRY, Brian; GOODING, Robert (eds.). Free movement: ethical issues in the transnational migration of people and money. Pennsylvania: Penn State University Press, 1992, p. 25-47.

CAVALLERO, Eric. An immigration pressure model of global distributive justice Politics. Philosophy \& Economics, v. 5, n. 1, 2006, p. 97-127.

COHEN, Gerald A. If you are an egalitarian, how come you're so rich? Cambridge: Harvard University Press, 2000.

COHEN, Gerald A. On the currency of egalitarian justice. Ethics, v. 99, n. 4, 1989, p. 906-944.

DWORKIN, Ronald. Equality, luck, and hierarchy. Philosophy \& Public Affairs, v. 31, n. 2, 2003, p. 190-198.

EASTERLY, William. The White Man's Burden: Why the West's Efforts to Aid the Rest Have Done So Much III and So Little Good. New York: Penguin Press, 2006.

GRIFFIN, James. Well-Being: Its Meaning, Measurement and Moral Importance. Oxford: Oxford University Press, 1986.

HARE, Richard M. Moral Thinking. Oxford: Oxford University Press, 1981.

HARSANYI, John. Essays on Ethics, Social Behavior, and Scientific Explanation. Reidel: Dordrecht, 1976.

HAYTER, Teresa. Open borders: the case against immigration controls. London: Pluto Press, 2000.

HÖFFE, Otfried. Demokratie in Zeitalter der Globalisierung. München: C.H. Beck, 1999.

KING, Timothy. Immigration from developing countries: some philosophical issues. Ethics, v. 93, n. 3, 1983, p. 525-536.

KYMLICKA, Will. Liberalism, community and culture. Oxford: Clarendon Press, 1989. KYMLICKA, Will. Multicultural citizenship. Oxford: Oxford University Press, 1995.

LEGRAIN, Philip. Immigrants: your country needs them. Princeton University Press, 2007.

LOEWE, Daniel. Los náufragos de nuestro tiempo. El caso de los refugiados. Arbor, v. 186, n. 744, 2010, p. 555-570.

LOEWE, Daniel. Cinco dimensiones del cosmopolitismo. Civitas, v. 15, n. 4, 2015, p. e155-e173.

LOEWE, Daniel. ¿Cuán liberal es la teoría de las relaciones internacionales de Rawls?. Veritas, v. 60, n. 1, 2015, p. e1-e35.

LOEWE, Daniel. Fronteras, Liberalismo e inmigración. Pensamiento, v. 72, n. 272, 2016, p. 633-654.

LOEWE, Daniel. Humanity's collective ownership of the earth and immigration. 2018 (Forthcoming).

LOEWE, Daniel. Inmigración y el derecho de gentes de John Rawls. Argumentos a favor de un derecho a movimiento sin fronteras. Revista de Ciencia Política, Santiago, v. 27, n. 2, 2007, p. 23-48. 
LOEWE, Daniel. Refugiados climáticos: ¿quién debe cargar los costos? REMHU, Revista Interdisciplinar da Mobilidade Humana, Brasília, Ano XXII, n. 43, 2014, p. 169-187.

MILANOVIC, Branko. Global inequality. A new approach for the age of globalization. Belknap Press, 2016.

MILLER, David. Citizenship and National Identity. Cambridge: Polity Press, 2000.

MILLER, David. Justice and inequality. In HURRELL, Andrew; WOOD, Ngaire (eds.). Inequality, globalization, and world politics. Oxford: Oxford University Press, 1999.

MILLER, David. National responsibility and global justice. Oxford: Oxford University Press, 2007.

MILLER, David. On Nationality. Oxford University Press, 1995.

NUSSBAUM, Martha. Frontiers of justice. Harvard: Harvard University Press, 2006.

POGGE, Thomas. Migration and poverty. In GOODIN, Roberto; PETTIT, Philip (eds.). Contemporary political philosophy: an anthology. Oxford: Blackwell, 2006, p. 710-720.

POGGE, Thomas. Realizing Rawls. Ithaca: Cornell University Press, 1989.

POGGE, Thomas. World poverty and human rights. Cambridge: Polity Press, 2002.

RAPOPORT, Hillel; DOCQUIER, Frédéric. The economics of migrants? Remittances. In KOLM, Serge-Christophe; YTHIER, Jean Mercier (eds.). Handbook on the economics of giving, reciprocity and altruism. North-Holland: Elsevier, 2006.

RAWLS, John. A theory of justice. Harvard: Harvard University Press, 1971.

RAWLS, John. Justice as fairness. KELLY, Erin (ed.). Cambridge Mass: Harvard University Press, 2001.

RAWLS, John. Political liberalism. New York: Columbia University Press, 1993.

RAWLS, John. The law of peoples with "The idea of public reason revisited". Harvard: Harvard University Press, 1999.

RAWLS, John. The law of peoples. In SHUTE, Stephen; HURLEY, Susan (eds.). On human rights: the Oxford amnesty lectures 1993. New York: Basic Books, 1993.

RISSE, Mathias. The Right to Relocation: Disappearing Island Nations and Common Ownership of the earth. Ethics \& International Affairs, v. 23, n. 3, 2009, p. 281300.

RISSE, Mathias. On the morality of Immigration. Ethics \& International Affairs, v. 22, n. 1, 2008.

RISSE, Mathias. Response to Arneson, de Bres, and Stilz. Ethics \& International Affairs, v. 28, n. 4, 2014, p. 511-522.

RISSE, Mathias. On Global Justice, Princeton: Princeton University Press, 2012.

SEGALL, Shlomi. Equality and opportunity. Oxford Uk.: Oxford University Press, 2013.

SHACHAR, Ayelet. The Birthright Lottery. Citizenship and Global Inequality. Mass.: Harvard University Press, 2009. 
SHEFFLER, Samuel. Equality and Tradition. New York: Oxford University Press, 2010.

SHEFFLER, Samuel. What is egalitarianism? Philosophy \& Public Affairs, v. 31, n. 1, 2003, p. 5-39.

SINGER, Peter. Famine, Affluence, and Morality. Philosophy and Public Affairs, v. 1, n. 3, 1972, p. 229-243.

SINGER, Peter. The life you can save. New York: Random House, 2009.

TAMIR, Yael. Liberal nationalism. Princeton: Princeton University Press, 1993.

TAN, Kok-Chor. Justice, institutions, and luck. Oxford UK: Oxford University Press, 2012.

VELASCO, Juan Carlos. El azar de las fronteras. Arroyo-México: Fondo de cultura económica, 2016.

WHELAN, Frederick. Citizenship and freedom of movement: an open admission policy? In GIBNEY, Mark (ed.). Open borders? Closed Societies? New York: Greenwood Press, 1992, p. 3-39.

WILCOX, Shelley. The open borders debate on immigration. Philosophy Compass, v. 4, n. 5, 2009, p. 813-821.

Recibido para publicación en 30.05.2017

Aceptado para publicación en 25.07.2017

Received for publication in May 30 ${ }^{\text {th }}, 2017$

Accepted for publication in July $25^{\text {th }}, 2017$

ISSN impresso 1980-8585

ISSN eletrônico 2237-9843

http://dx.doi.org/10.1590/1980-85852503880005003 